\title{
Topical issue: New trends in porous media
}

The recent development of "visualization" techniques in porous media, such as 3D X-ray micro-tomography and nuclear magnetic resonance (NMR), leads not only to characterize more accurately the structure of porous media but to follow its evolution under external constraints.

To illustrate these new trends in porous media, we choose five articles focusing mainly on the new "visualization" techniques and the related ability to follow the evolution to these materials at small scales:

- The first article, using X-ray micro-tomography, addresses the evolution of the topology of the pore networks during growth or aging.

- The second one, using also 3D X-ray micro-tomography, deals with the micro-fracturation process in rocks.

- In the third one, NMR is used to revisit the dynamics of hydrodynamic dispersion.

- NMR imaging, used to follow the damage resulting from the effect of salt crystallization, is the focus of the fourth article.

- The last article, thanks to X-ray computer tomography, analyzes the alteration of porous media due to the drying of brines.

These five articles, hopefully, will convince that development of techniques is a key issue to gain a better understanding of the processes at work inside porous media.

Dominique Salin

Université Pierre et Marie Curie (Paris)

Editor of The European Physical Journal Applied Physics 